\title{
Nature-inspired novel Cuckoo Search Algorithm for genome sequence assembly
}

\author{
R INDUMATHY $^{1, *}$, S UMA MAHESWARI ${ }^{1}$ and G SUBASHINI ${ }^{2}$ \\ ${ }^{1}$ Department of Electronics and Communication Engineering, Coimbatore Institute \\ of Technology, Coimbatore 641 014, India \\ ${ }^{2}$ Department of Information Technology, PSG College of Technology, \\ Coimbatore 641 004, India \\ e-mail: indumathy.rajagopal@gmail.com; umamaheswari.cit@gmail.com; \\ suba@ity.psgtech.ac.in
}

MS received 1 May 2013; revised 28 January 2014; accepted 24 August 2014

\begin{abstract}
This study aims to produce a novel optimization algorithm, called the Cuckoo Search Algorithm (CS), for solving the genome sequence assembly problem. Assembly of genome sequence is a technique that attempts to rebuild the target sequence from the collection of fragments. This study is the first application of the CS for DNA sequence assembly problem in the literature. The algorithm is based on the levy flight behaviour and brood parasitic behaviour. The CS algorithm is employed to maximize the overlap score by reconstructing the original DNA sequence. Experimental results show the ability of the CS to find better optimal genome assembly. To check the efficiency of the proposed technique the results of the CS is compared with one of the well known evolutionary algorithms namely, particle swarm optimization (PSO) and its variants.
\end{abstract}

Keywords. Bioinformatics; Cuckoo search; genome sequence assembly; metaheuristics

\section{Introduction}

The innovation in the cutting edge of soft computing technology proposes solutions to solve many challenging bioinformatics problems. A heuristic optimization technique has been applied for most exciting research areas in computational biology. One of the major research areas in the genomics field is the genome sequence assembly. The shotgun sequencing approach has been used broadly in genome sequencing projects. The process of genome sequence assembly begins by breaking the DNA into millions of random fragments and reconstruct the fragments to obtain a longer consensus sequence. This being an NP-hard combinatorial optimization problem,

${ }^{*}$ For correspondence 
which is increasing in significance and complexity, heuristic and meta-heuristic procedures can be applied to this approach to assemble the fragments because finding the exact order takes a huge amount of time. This is the challenging task in any genome project.

\section{Related work}

Many literatures provide solutions to genome sequence assembly. Christian Burks (1994) suggested that DNA sequencing throughput would be increased by orders of magnitude to complete the task in a period of 15 years that was laid out for the Human Genome Project. Many heuristic approaches are applied in DNA Sequence Assembly to improve the process of DNA Sequence Assembly. One of them is Genetic Algorithm. Parsons et al (1995) analysed various genetic algorithm operators for one permutation problem related with the Human Genome Project. Parson \& Johnson (1995) discussed the modifications in the previous genetic algorithm used, the experimental design process by which new results were obtained and have also made preliminary attempts to explain the results and to obtain proper solution. Kim \& Mohan (2003) present a fragment assembler using a new parallel hierarchical adaptive variation of evolutionary algorithms. The approach of Fang et al (2005) maximizes the similarity (overlaps) between given fragments and a candidate sequence.

Luque \& Alba (2005) presented several methods: a canonical genetic algorithm, a cross generational elitist selection method, a scatter search algorithm, and a simulated annealing, to solve the problem accurately for instances that are $77 \mathrm{~K}$ base pairs long. Kikuchi \& Chakraborty (2006) added two heuristic ideas with GA to make it more efficient. The first step is Chromosome Reduction (CRed) step and the second step is Chromosome Refinement (CRef) step. Nebro et al (2008) proposed a grid based genetic algorithm in solving the DNA fragment assembly problem. Enrique \& Gabriel (2008) solved the DNA fragment assembly problem by using a new hybrid genetic algorithm.

A number of fragment assembly packages have been developed which were used to sequence different organisms. The widely used popular package PHRAP Green (1997) is a program for assembling shotgun DNA sequence data. The TIGR assembler (Sutton et al 1995) overcomes several major obstacles in assembling projects such as: the large number of pair-wise comparisons required for the presence of repeat regions, chimeras introduced in the cloning process and sequencing errors. Chen \& Skiena (1998) implemented a reliable technique to sequence DNA using primer-walking approach. CAP3 (Huang \& Madan 1999) program includes a number of optimizations and new features to improve DNA sequence assembly. Myers (2000) developed Celera assembler at Celera for publication of the first draft human genome sequence in 2001. Pevzner (2000) suggested a novel approach to fragment assembly that abandons the classical 'overlap-layout-consensus' pattern and was used in all currently available assembly tools.

Meksangsouy \& Chaiyaratana (2003) proposed an asymmetric ordering representation where a path, co-operatively generated by all ants in the colony represents the search solution. Zhao et al (2008) improved sequence alignment method based on the ant colony algorithm. Zuwairie \& Kurniawan (2009) in their approach model represented the DNA sequence design as a pathfinding problem, which consists of four nodes, to enable the implementation of the ACS and compared their results with other methods such as the genetic algorithm. Ravi et al (2011) proposed a solution for DNA sequence assembly problem using Particle Swarm Optimization (PSO) with Shortest Position Value (SPV) rule. Elloumi \& Kaabi (1999) proposed one of the optimization algorithms for the fragment assembly problem is the Greedy Algorithm, based on the best set of Maximum Weight Contigs Approach. 


\section{Genome sequence assembly}

Genome sequence comprises entirely of Deoxyribonucleic acid (DNA) which is a nucleic acid that contains the genetic information used in the development and functioning of all known living organisms and some viruses. DNA sequence is made of four nucleotides namely, Adenine (A), Thymine (T), Guanine (G) and Cytosine (C). A DNA molecule comprises of two parallel strands consisting of these nucleotides in the forward and reverse orientation. The nucleotides exhibit a complementary relationship with other nucleotides such that the two strands are held together through bonding between complementary nucleotides. For instance, Adenine on one strand binds to Thymine with two hydrogen bonds on the opposite strands and similarly Cytosine binds to Guanine with three hydrogen bonds. This bonding gives rise to the renowned double helical structure for DNA. The size of the human genome consists of 23 chromosome pairs, which is about 3.2 billion nucleotides in length and cannot be read at once. Sophisticated sequence assembly algorithms are needed to efficiently perform the assembling process. The DNA sequence assembly for shotgun sequencing is performed by cloning the DNA sequence into several copies, splitting each copy into millions of random fragments, reading the individual fragments and then assembling those fragments. The process of assembling huge sequences can be divided into two major steps. The first step is to rebuild the longer sequence from the short fragments by using heuristic algorithms. The second step is to align the sequence obtained from the assembling process through sequence alignment algorithms. One of the genome sequence assembly procedures is the Overlap-Layout Consensus approach.

Overlap Stage: Finding the overlapping fragments deals with the comparison of all possible pairs of fragments using semi global alignment algorithm to determine the similarity score.

Layout Stage: Finding the exact order of fragments based on computed similarity scores. This stage is complicated because it is tough to decide accurate overlaps.

Consensus Stage: Deriving the DNA sequence from the layout stage. The common technique used in this stage is to apply the majority rule in building the consensus.

An illustration of genome sequence assembly technique is shown in figure 1. (a) Given a set of four fragments, (b) to find the overlap, (c) to find the layout and (d) to assemble the four fragments into the consensus sequence.

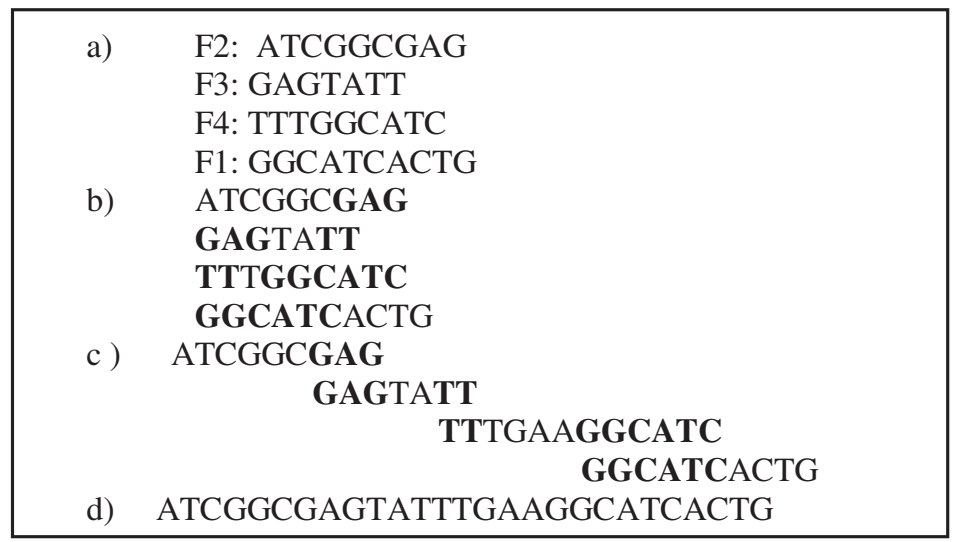

Figure 1. Example of genome sequence assembly technique. 
Since finding the exact order takes a huge amount of time, a heuristic technique can be applied in this step. The quality of a consensus sequence is normally measured by the term coverage. It is defined as the total number of base reads from fragments as a ratio of the length of the source DNA :

$$
\text { Coverage }=\frac{\sum_{i=0}^{n} \text { Length of the Fragment i }}{\text { Target Sequence Length }},
$$

where $n$ is the total number of fragments. If coverage is high, the probability of covering original genome is higher and the correctness of the assembled parts has been enhanced. Coverage (Setubal \& Meidanis 1997) is evaluated according to equation (1).

\section{Overview of cuckoo search algorithm}

Cuckoo search algorithm is one of the most recently defined meta-heuristic algorithms proposed by Yang \& Deb (2009, 2010). It has been developed by simulating the intelligent breeding behaviour of cuckoos. It is a population-based search procedure used as an optimization tool, in solving complex optimization problems. Cuckoos lay their eggs in the nests of other host birds with incredible abilities like selecting the recently spawned nests and eliminating existing eggs that enhance hatching probability of their eggs. The host bird takes care of the eggs presuming that the eggs are its own. However, some of host birds are able to combat with this parasitic behaviour of Cuckoos, and throw out the identified alien eggs or build their new nests in new locations. Each egg in a nest represents a solution, and a Cuckoo's egg represents a new solution. When generating a new solution Levy flight is performed. The three ideal rules for CS are described as follows:

(i) Each Cuckoo lays one egg at a time, and deposits it in a randomly chosen nest.

(ii) The best nests with high quality of eggs will carry over to the next generations.

(iii) The number of available host nests is fixed and there is a probability that a host can discover an alien egg. In this case, the host bird can either throw the egg away or abandon the nest to build a completely new nest in a new location.

A randomly distributed initial population of $\mathrm{M}=\left[\mathrm{X}_{1} ; \mathrm{X}_{2} ; \mathrm{X}_{3} ; \ldots ; \mathrm{X}_{\mathrm{m}}\right]$ solutions or host nest positions in the multi-dimensional host nest is generated. Each solution $\mathrm{X}$ is represented by the D-dimensional vector. The Cuckoo randomly chooses the host nest position to lay an egg using Levy flights random walk and is given in Eqs. (2) and (3).

$$
\begin{gathered}
V_{p q}^{t+1}=V_{p q}^{t}+S_{p q} * \operatorname{Levy}(\lambda) * \alpha \\
\operatorname{Levy}(\lambda)=\left|\frac{\Gamma(1+\lambda) * \sin \left(\frac{\Pi * \lambda}{2}\right)}{\Gamma\left(\frac{(1+\lambda)}{2}\right) * \lambda * 2 \frac{(\lambda-1)}{2}}\right|^{\frac{1}{\lambda}},
\end{gathered}
$$

where $p, f \in\{1,2, \ldots, m\}$ and $q \in\{1,2, \ldots, D\}$ are randomly chosen indexes from $p . D$ is the number of parameters to be optimized and $m$ is the total population of host nest positions. $\lambda$ is a constant $(1 \leq \lambda \leq 3), t$ is the current generation number and $\alpha$ is a random number generated between $[-1,1]$. Also, $s>0$ is the step size. If $s$ is too large, then the new solution generated will be too far away from the old solution. If it's too small, the change is too small to be significant 
and consequently such search is not efficient. So a proper step size is important to maintain the search as efficiently as possible. Hence, the step size is calculated using Eq. (4).

$$
S_{p q}=V_{p q}^{t}-V_{f q}^{t} .
$$

Using Eq. (3) the Cuckoo chooses the host nest and an egg laid by a Cuckoo is evaluated. The host bird identifies the alien egg with the probability value associated with that quality of an egg using Eq. (5).

$$
\text { Pro } o_{p}=(0.9 * F(I) / \max (F i t))+0.1,
$$

where $F(I)$ is the fitness value of the solution $p$ which is proportional to the quality of an egg in the nest position $p$ and Pro $_{p}$ gives the survival probability rate of the Cuckoo's egg. If the random generated probability $\mathrm{P}_{\mathrm{a}} \in[0,1]$ is greater than the Pro $\mathrm{p}_{\mathrm{p}}$, then the alien egg is identified by the host bird. Then the host bird, destroys the alien egg away or abandon the nest and, Cuckoos find a new host's nest (in new position) using Eq. (6) for laying an egg. Otherwise, the egg grows up and be alive for the next generation based on the fitness function.

$$
X_{p}=X_{p_{\min }}+\operatorname{rand}(0,1) *\left(X_{p_{\max }}-X_{p_{\min }}\right),
$$

where $X_{p_{\min }}$ and $X_{p_{\max }}$ are the minimum and maximum limits of the parameters to be optimized as proposed by Chandrasekaran \& Simon (2012).

The pseudo code of the CS algorithm is given below:

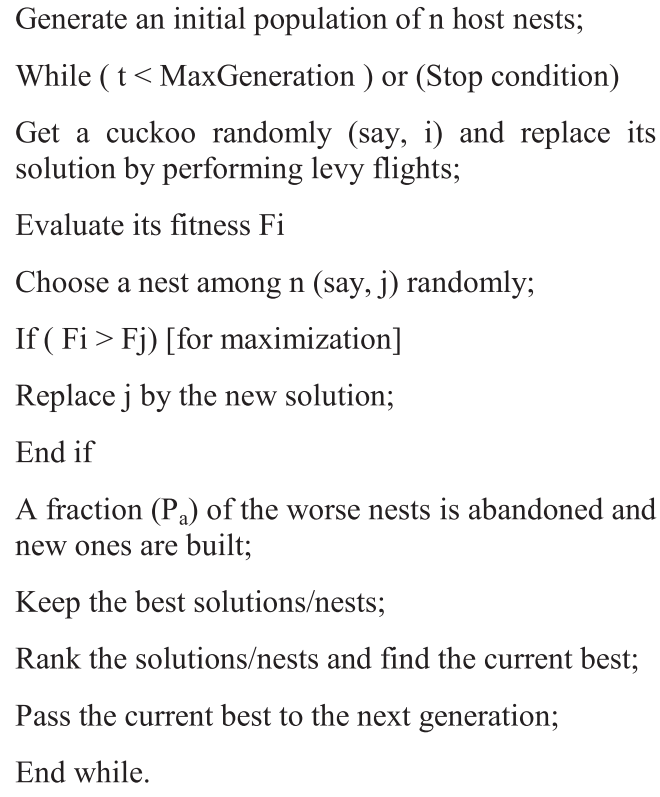

\section{Genome sequence assembly using cuckoo search algorithm}

In this section, the problem formulation, fitness evaluation, flowchart and implementation of the proposed technique discussed. 


\subsection{Problem formulation}

The problem formulation is the initial step in any optimization problem. Genome sequence assembly has been performed according to cuckoo search algorithm. A genome is cloned and randomly cut into different fragments of varied length. The input to this optimization problem is the set of fragments. The fragments are aligned and the matching nucleotide score is evaluated using semi global alignment algorithm. Permutation of fragment are generated and all possible pair combination of fragments are compared then the matching scores (fitness) is evaluated. The fragments are then assembled to form a consensus sequence without any repeated patterns. Finally, consensus sequence is formed from the fragment order.

In Cuckoo search algorithm, the order of the fragments is determined. Cuckoo search is a population based search procedure; here each nest represents a solution for a problem. In genome sequence assembly each of the nest represents a permutation of fragments of a DNA sequence. The position of the nest represents a possible solution. Each solution has certain dimension value equal to the number of fragments taken for assembly. Real coded Cuckoo search algorithm has been used for solving the genome sequence assembly problem.

\subsection{Fitness evaluation}

A fitness function is calculated after solution representation. Parsons et al (1995) proposed the fitness function as shown in Eq. (7). The fitness function, $F(I)$, sums the overlap score for adjacent fragments $\mathrm{F}[\mathrm{i}]$ and $\mathrm{F}[\mathrm{i}+1$ ] in a given solution. The objective function is a maximization function that involves maximizing the score. It means that the best individual will have the highest score.

$$
F(I)=\sum_{i=0}^{n-2} W(F[i], F[i+1]),
$$

where $\mathrm{I}$ is the individual and $W(\mathrm{~F}[i], \mathrm{F}[i+1)$ is the pair-wise overlap strength of fragments $\mathrm{F}[i]$ and $\mathrm{F}[i+1]$. The overlap score $(W)$ in $\mathrm{F}$ is computed using the semi global alignment algorithm, a classical dynamic programming technique to calculate the pair-wise alignments. The example of semi-global alignment is shown in figure 2. For the input DNA Sequence S1: ATCG and S2: ATTCG, the match score is defined by 2, a mismatch score is defined by 0 , and a penalty of gap defined by - 2. Finally, the overlap score between S1 and S2 was calculated to be 6, and the new S1 is modified in AT-CG, the symbol "-" represents gap in a DNA sequence assembly. However, the best individual would get the highest score value between the population.

\subsection{Implementation of real coded CS algorithm for genome sequence assembly}

Cuckoo search algorithm is the proposed technique used to solve the genome sequence assembly problem. In the problem of DNA sequence assembly the inputs are set to different lengths of fragments, the target is to generate permutation of fragments and to obtain the maximum fitness to build the consensus sequence which should not have any repeated patterns. Solution representation is the initial step of the Cuckoo search algorithm. Each fragment is represented by real values. The subsequent step begins by generating the initial host nest population randomly. Using Eq. (2) the fragment position is modified by using levy flights random walk. The fitness has been evaluated for the generated fragment positions using Eq. (7). Randomly selected another sequence and the fitness is calculated. The sequences with worst fitness scores have been discovered and abandoned using Eq. (5). Then the new host nest population (sequences) is generated 


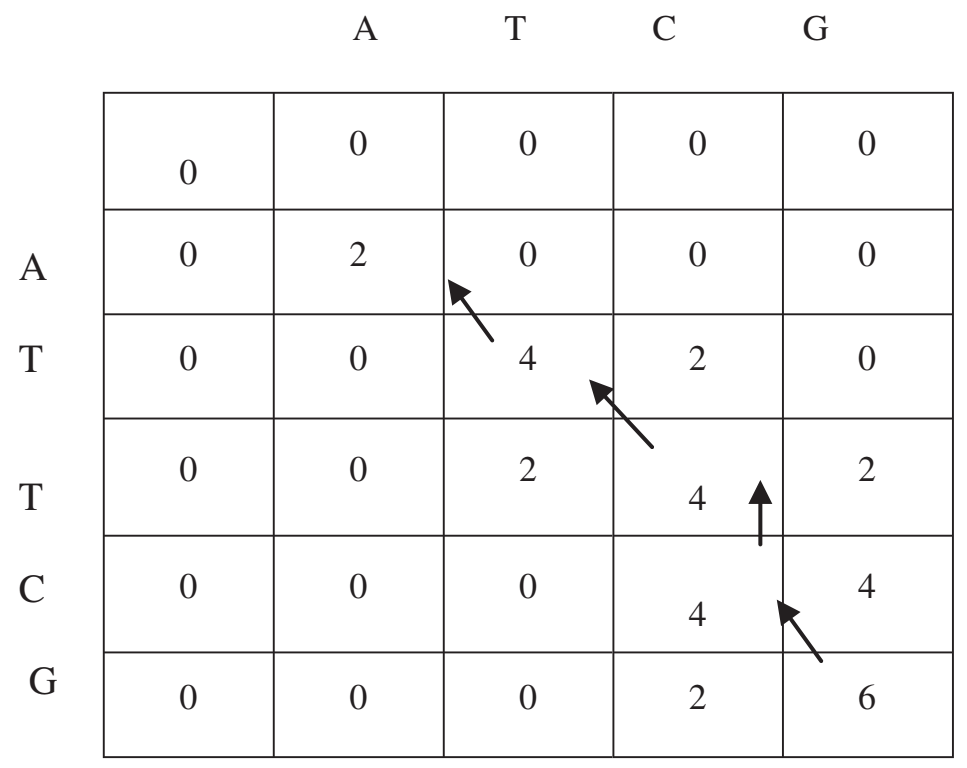

Figure 2. Examples of semi-global alignment algorithm.

using Eq. (6).The fitness for the generated new sequences was evaluated. Finally, the consensus DNA sequence was computed. This process was terminated by specifying the maximum number of generations reached or when the solution is no longer improving. The above procedure is described using flowchart in figure 3.

\section{Results and discussion}

In this section, the experimental set-up and the performance of the proposed method were analysed. The problem instances that have been generated by Engle \& Burks (1993). GenFrag was used in this assessment. GenFrag takes a known sequence and uses it as a parent strand from which random fragments were generated according to the criteria given by the user. The two major conditions were mean fragment length and coverage of parent sequence. To test the performance of the proposed method, three sequences have been chosen from the National Center for Biotechnology Information (NCBI). Table 1 presents information about the specific fragment sets used to evaluate the proposed method.

The experiments have been executed on a Dell XPS L502X with Intel (R) Core(TM) i7 2670 QM CPU @ $2.20 \mathrm{GHz}$ with 8 GB of RAM running on Microsoft Windows 7 platform. The proposed algorithm is implemented using C\# programming language on the .NET platform and the database used is MySQL. The proposed method implementations are simulated 30 times with different random seeds and their average fitness scores were recorded. The cuckoo search algorithm parameter settings were selected as shown in table 2.

To validate and analyse the performance of the proposed algorithm the overlap score of the nucleotides were evaluated. The fitness function was used to calculate the overlapping score of adjacent fragments. The major objective is to maximize this total score. This paper discusses the evaluation of overlapping score of the proposed algorithm. The impact of the overlap score calculation is to ensure that the best solution represents an assembled consensus sequence. The 


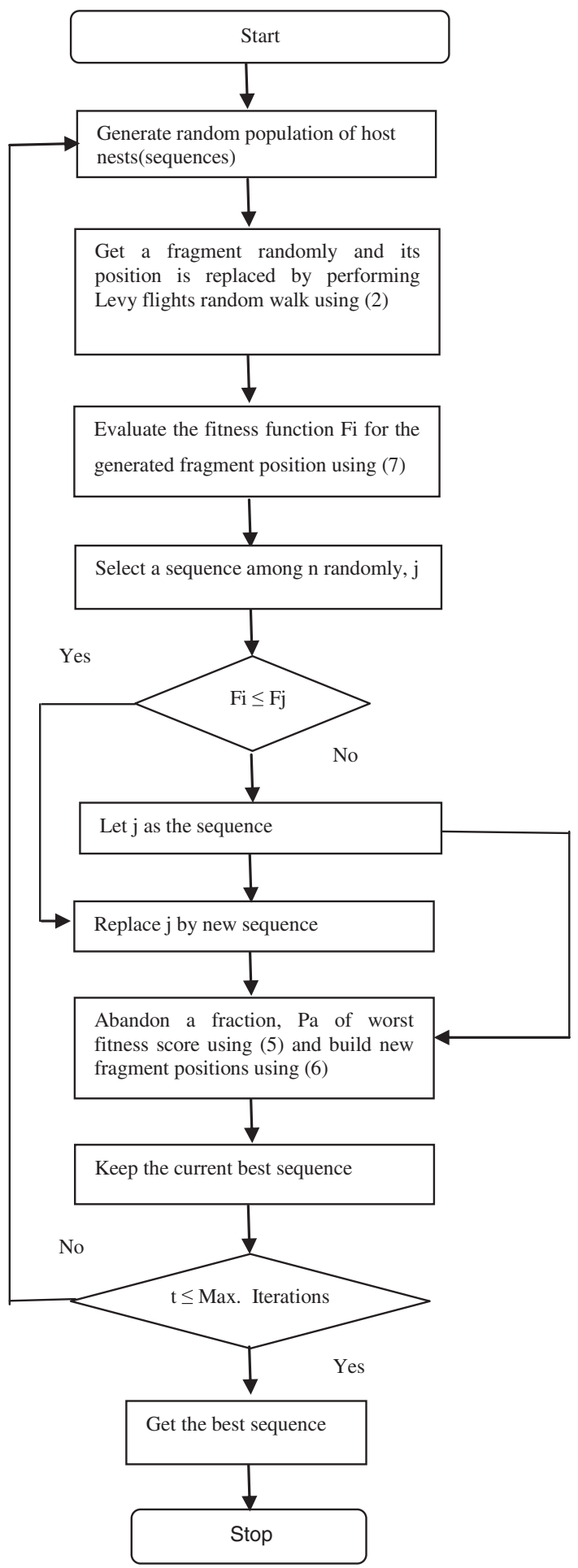

Figure 3. Flowchart of the proposed Cuckoo search algorithm. 
Table 1. The GenFrag Benchmarks for DNA sequence assembly problem.

\begin{tabular}{lccc}
\hline Accession no. & Sequence length (bps) & Number of fragments & Coverage \\
\hline X60189(4) & 3,835 & 39 & 4 \\
X60189(5) & & 48 & 5 \\
X60189(6) & & 66 & 6 \\
X60189(7) & & 68 & 7 \\
M15421(5) & 10,089 & 127 & 5 \\
M15421(6) & & 173 & 6 \\
M15421(7) & \multirow{2}{*}{20,100} & 177 & 7 \\
J02459(7) & & 352 & 7 \\
\hline
\end{tabular}

efficiency of the proposed algorithm has been compared with other meta-heuristic optimization techniques called the Particle Swarm Optimization (PSO), Adaptive Particle Swarm Optimization (APSO) and Constriction Factor Particle Swarm Optimization (CPSO). PSO is based on the concept of population and each individual represents a solution to a problem. The matching nucleotide score (fitness value) is the only measure to assess the quality of the solution. Table 3 provides the comparison of the overlap score obtained from real coded Cuckoo search with respect to other available techniques called particle swarm optimization and its variants.

The experimental results clearly demonstrate the efficiency and accuracy of the proposed CS technique than the other technique. The CS parameters help to enhance the convergence rate. The three basic control parameters namely, initial population of the host nests, maximum generation limit and $\lambda$ have been optimally tuned. The variation of CS parameters can yield better solution and reduce the computational time. The overlapping scores were compared by increasing number of iterations and by varying the nest population size. The total number of evaluation is hundred. For the data instance X60189(4) with the nest population size of 20, in the CS method the overlap score obtained initially was 11810 . As illustrated in figure 4, the overlap score increases when the number of iterations increases and it converges to the maximum overlap score of 12933 at the $70^{\text {th }}$ iteration and remains the same until the last iteration. In PSO method, the overlap score obtained initially was 11588 and when the number of iterations increased the overlap score reached to 12581 at $60^{\text {th }}$ iteration and remained the same until the last iteration. The average value for 30 trials with different random seeds was recorded. However, the CS method got the maximum overlap score when compared with PSO and its variants.

When there is an increase in the nest population size, the number of iterations required by the Cuckoo search algorithm to converge to the optimum solution decreases. As illustrated in figure 5, for the data instance M15421 (5) the maximum overlap scores were obtained by varying the nest population size. An increase in the nest population size leads to an improvement in the average overlap score. The parameter $\lambda$ has been varied from one to three to obtain the optimum solution. The maximum number of iterations for nest population size 10 to 50 ranges from 20

Table 2. Cuckoo search parameters.

\begin{tabular}{lc}
\hline Parameter description & Parameter value \\
\hline Population size & 20,50 \\
Number of generations & 100 \\
$\lambda$ & {$[1,3]$} \\
$\alpha$ & {$[-1,1]$} \\
\hline
\end{tabular}


Table 3. Experimental results of the CS against PSO and its variants for population size $=50$.

\begin{tabular}{|c|c|c|c|c|c|}
\hline \multirow[t]{2}{*}{ SL. No. } & \multirow[t]{2}{*}{ Name of the instance } & \multicolumn{4}{|c|}{ Overlap score } \\
\hline & & PSO & APSO & CPSO & $\mathrm{CS}$ \\
\hline 1 & X60189(4) & 12476 & 12603 & 12669 & 12703 \\
\hline 2 & X60189(5) & 14552 & 14726 & 14991 & 15082 \\
\hline 3 & X60189(6) & 17412 & 17815 & 17923 & 18349 \\
\hline 4 & X60189(7) & 20197 & 20218 & 20386 & 20394 \\
\hline 5 & M15421(5) & 36995 & 37582 & 37504 & 38228 \\
\hline 6 & M15421(6) & 45816 & 45874 & 46459 & 46811 \\
\hline 7 & M15421(7) & 49096 & 49492 & 49893 & 50738 \\
\hline 8 & $\mathrm{~J} 02459(7)$ & 102128 & 103202 & 105658 & 105942 \\
\hline
\end{tabular}

to 100 iterations. Hence, the parameter settings play a major role in the convergence rate and it produces quality solutions when compared with the other techniques.

For the data instance X60189(7) with the nest population size of 50, in the CS method the overlap score obtained initially was 19006. As illustrated in figure 6, the overlap score increases when the number of iterations increases and it converges to the maximum overlap score of 20394 at $80^{\text {th }}$ iteration and remains the same until the last iteration. In standard PSO method the overlap score obtained initially was 18990 and when the number of iterations increases the overlap score reaches to 20197 at $90^{\text {th }}$ iteration and remains the same until the last iteration. The average value for 30 trials with different random seeds was recorded. However, the Cuckoo search technique gets the maximum overlap score when compared with PSO technique and its variants. For the real coded Cuckoo search algorithm the best combination of parameter settings are $\lambda=1.5$, initial population of the host nest $=50$ and the maximum number of iteration limit $=100$. The

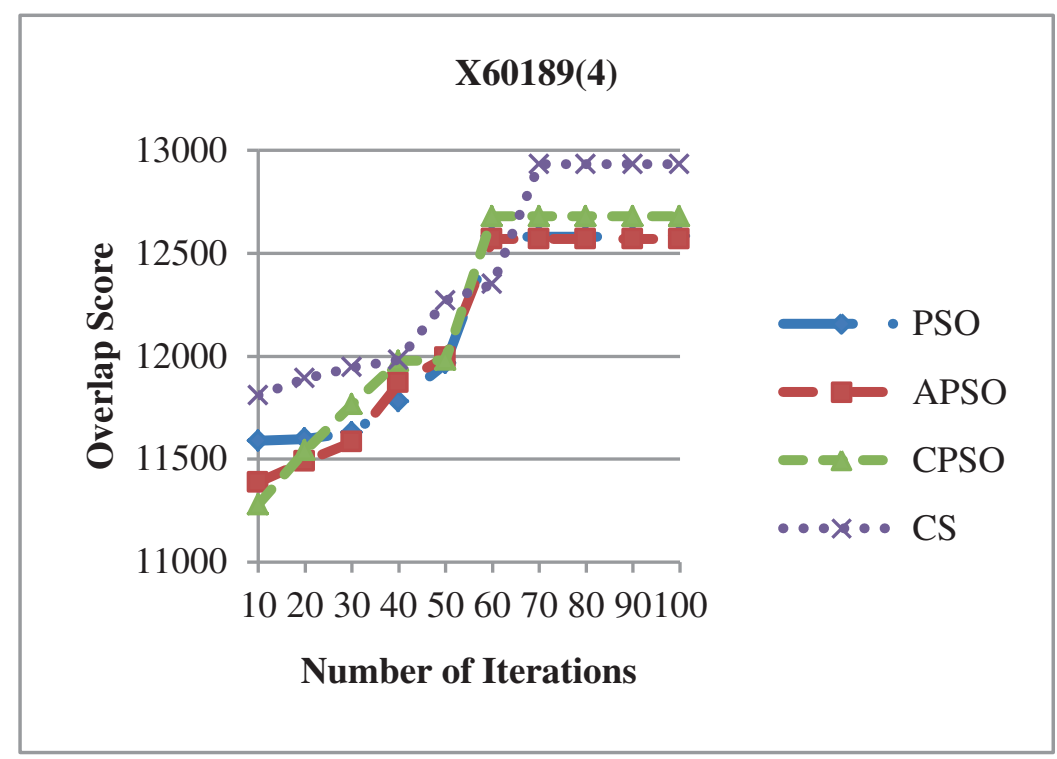

Figure 4. Comparison graph for CS algorithm and PSO algorithm for the benchmark dataset X60189(4) by varying the number of iterations for population size $=20$. 


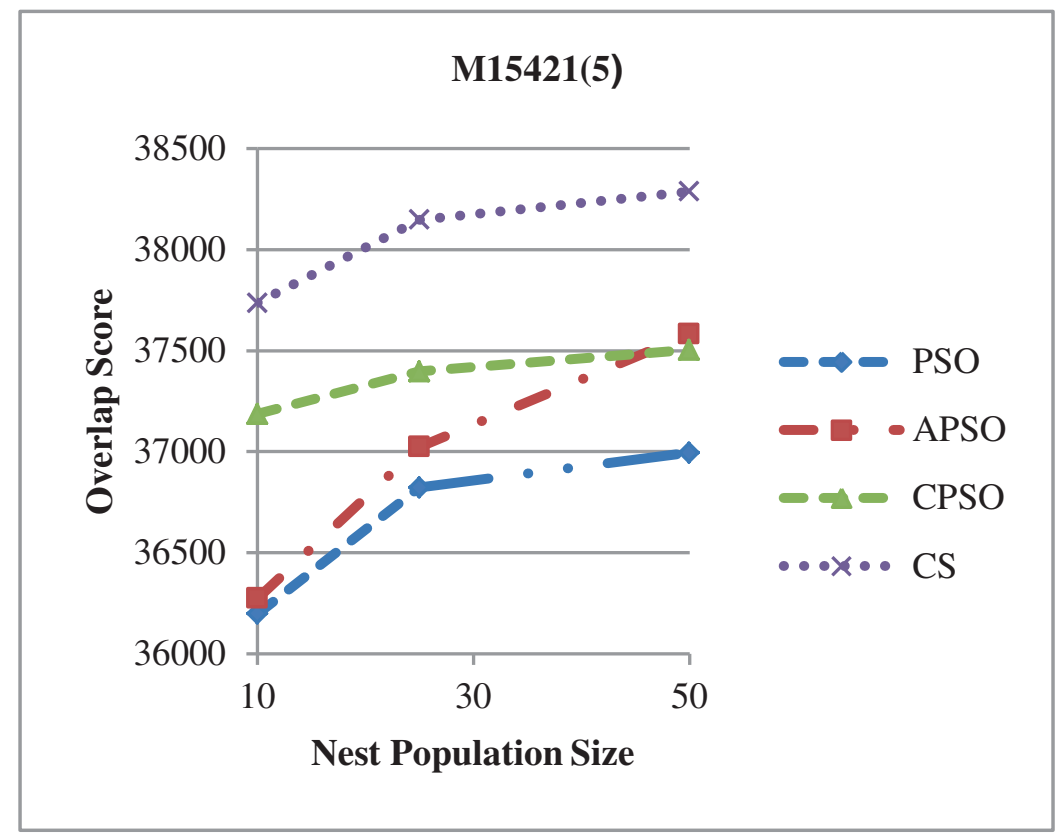

Figure 5. Comparison graph for CS algorithm, PSO algorithm and its variants for the benchmark dataset M15421(5) by varying the population size.

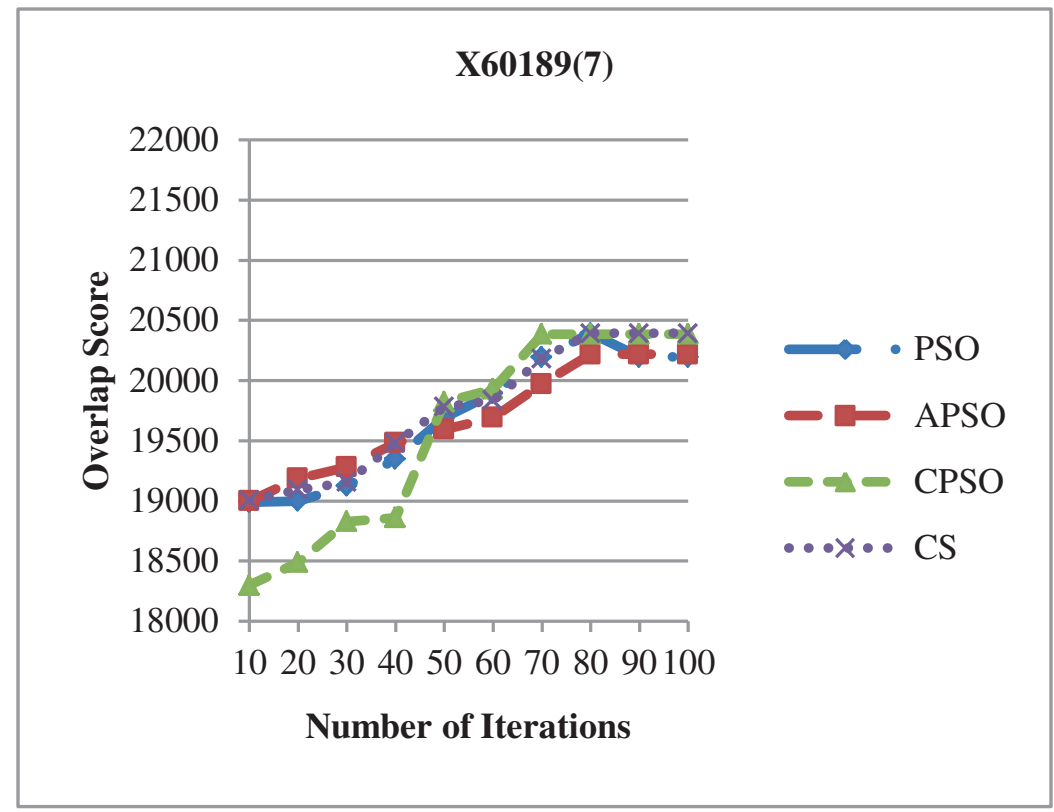

Figure 6. Comparison graph for CS algorithm, PSO algorithm and its variants for the benchmark dataset $\mathrm{X} 60189$ (7) by varying the number of iterations for population size $=50$. 


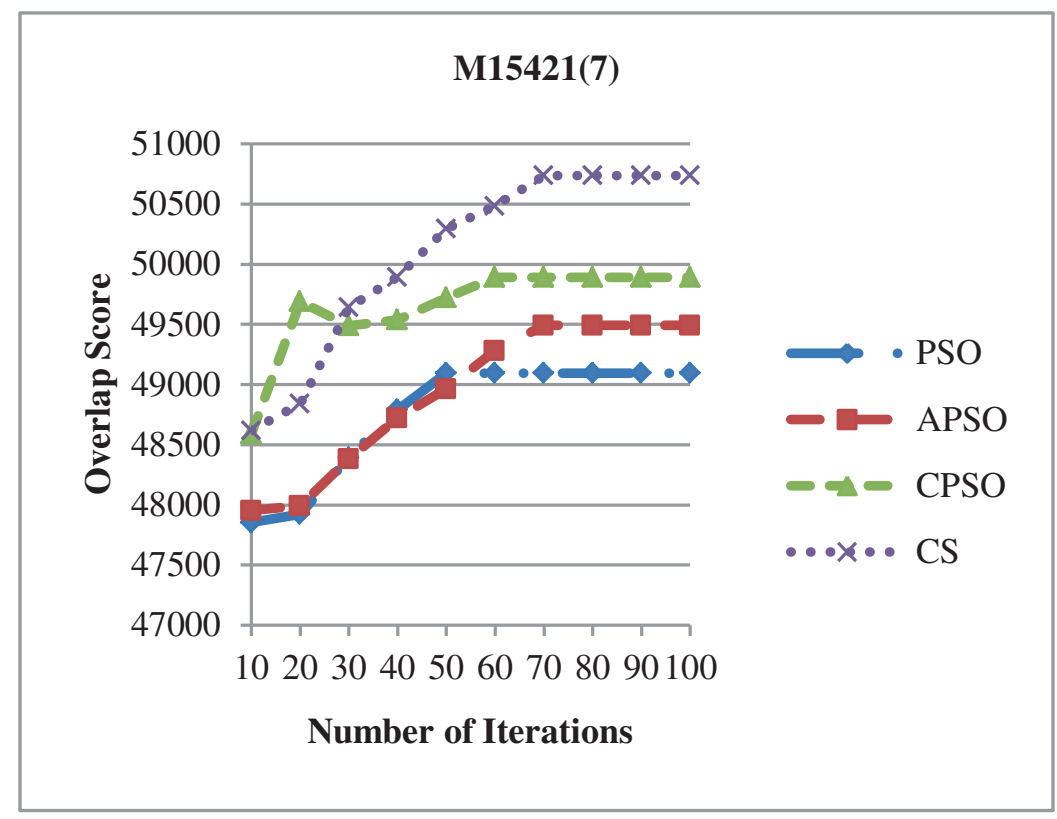

Figure 7. Comparison graph for CS algorithm, PSO algorithm and its variants for the benchmark dataset M15421 (7) by varying the number of iterations for population size $=50$.

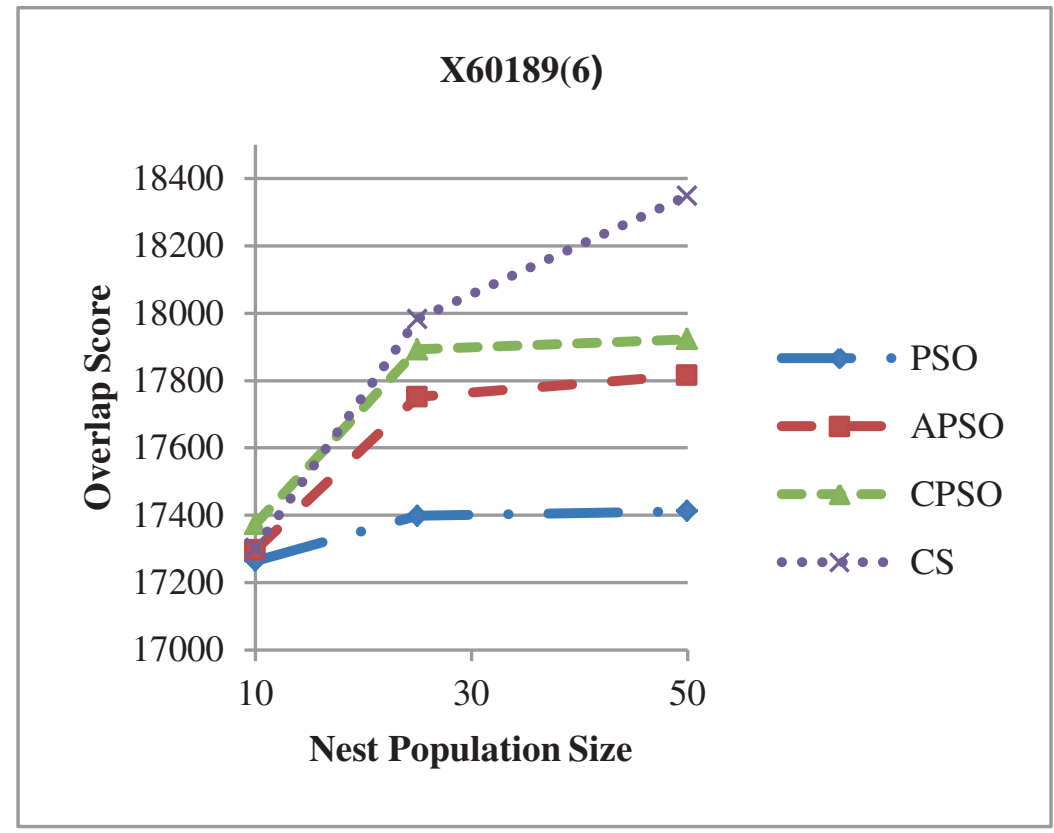

Figure 8. Comparison graph for CS algorithm, PSO algorithm and its variants for the benchmark dataset $\mathrm{X} 60189(6)$ by varying the nest population size. 
parameter tuning is carried out efficiently to find out the final combination of parameters that helps to provide the optimal solution.

For the data instance M15421 (7) with the nest population size of 50, in the CS technique the overlap score obtained initially was 48620 . As shown in figure 7 , the convergence occurs at lower number of iterations with a gradual and slight increase in the overlap score, which reaches up to 50738, when the number of iterations increases. Whereas, the overlap score is less for PSO technique and its variants when compared to the CS technique.

As illustrated in figure 8, for the data instance X60189(6) the maximum overlap scores were obtained by varying the nest population size. In CS technique for the population size 10 and the iteration limit was set to 50, the average overlap score was 17308 . When the population size was 25 and the iteration limit was set to 50 the average overlap score was found to be 18349 . In PSO technique and its variants there was an improvement in the average overlap score when the nest population size was increased. Hence, an increase in the nest population size leads to an improvement in the average overlap score. The maximum number of iterations for nest population size 10 to 50 ranges from 20 to 100 iterations. Hence, the parameter settings play a major role in the convergence rate and it produces quality solutions when compared with the other techniques.

\section{Conclusion}

The genome sequence assembly is a NP-hard problem that attempts to rebuild the target sequence from the collection of fragments. This research analysis has presented a novel Cuckoo search algorithm using Levy flight for the genome sequence assembly problem. The search behaviour of Cuckoos for laying an egg in the host nest is modelled and used for solving the genome sequence assembly problem. Computational techniques have been applied to obtain an optimum solution. CS algorithm seeks the most improving modification of the current solution in each iteration. Different algorithm settings of the CS algorithm have been analysed to obtain the optimal solution. Genome sequence assembly problem considered in this paper is to maximize the overlapping score. The performance of the proposed CS based algorithm, PSO algorithm and its variants has been validated on the different GenFrag benchmark data instances. It has been shown through different trials that the Cuckoo search method outperforms the PSO method and its variants in terms of high quality solution, consistency, faster convergence, and accuracy. The performance of the proposed CS algorithm is found to be better when compared with PSO, APSO and CPSO algorithms.

In the forthcoming research work, efficient strategies can be employed for enhancing the algorithm to obtain better overlap score. Various nature-inspired algorithms can be applied to solve the complicated genome sequence assembly problem and the results can be compared with CS algorithm. Hybridization of algorithms with the proposed CS algorithm may give enhanced results than the existing nature-inspired algorithms. The various variants of CS algorithm can be implemented to solve the genome sequence assembly problem.

\section{Acknowledgements}

We thank the Coimbatore Institute of Technology, Coimbatore for providing us necessary facilities and support. We are thankful to Dr. Raja C Mugasimangalam, Genome Informatics team and Microarray Design Team at Genotypic Technology Private Limited, Bangalore, for the helpful discussions on genome fragment assembly. 


\section{References}

Burks C 1994 DNA sequence assembly. Engineering in Medicine and Biology Magazine. IEEE 13: 771773

Chandrasekaran K and Simon S P 2012 Multi-objective scheduling problem: Hybrid approach using fuzzy assisted cuckoo search algorithm. Swarm and Evolutionary compution 5: 1-16

Chen T and Skiena S 1998 Tree-based data structures for sequence assembly. Combinatorial Pattern Matching 206-223

Elloumi M and Kaabi S 1999 Exact and approximation algorithms for the DNA sequence assembly problem. SCI in Biology and Medicine 8

Engle M L and Burks C 1993 Artificially generated data sets for testing dna sequence assembly algorithms. Genomics 16(1): 286-288

Enrique Alba and Gabriel Luque 2008 A Hybrid Genetic Algorithm for the DNA Fragment Assembly Problem. Recent advances in Evolutionary Computation for combinatorial optimization Studies in computational Intelligence, Springer, 153: 101-112

Fang S C, Wang Y and Zhong J 2005 A Genetic Algorithm Approach to Solving DNA Fragment Assembly Problem. J. Computational and Theoretical Nanoscience 2: 499-505

Green P 1997 Against a whole genome shotgun. Genome Research 7: 410-417

Huang X and Madan A 1999 CAP3: A DNA sequence assembly program. Genome Res 9: 868-877

Kikuchi S and Chakraborty G 2006 Heuristically tuned GA to solve genome fragment assembly problem. Evolutionary Computation. IEEE: 1491-1498

Kim K and Mohan C K 2003 Parallel hierarchical adaptive genetic algorithm for fragment assembly. Evolutionary Computation, CEC'03, 1: 600-607

Luque G and Alba E 2005 Metaheuristics for the DNA fragment assembly problem. Int. J. Comput. Intelligence Res. 1: 98-108

Meksangsouy P and Chaiyaratana N 2003 DNA fragment assembly using an ant colony system algorithm. Evolutionary Computation 3: 1756-1763

Myers E W 2000 Towards simplifying and accurately formulating fragment assembly. J. Computational Biol. 2: 275-290

Nebro A J, Luque G, Luna F and Alba E 2008 DNA Fragment assembly using a grid-based algorithm. Computers and Operations Res. 35: 2776-2790

Parsons R J and Johnson M E 1995 DNA sequence assembly and genetic algorithms- new results and puzzling insights. Proceedings of the Third International Conference on Intelligent Systems for Molecular Biology (ISMB-95), 277-284

Parsons R J, Forrest S and Burks C 1995 Genetic algorithms, operators, and DNA fragment assembly. Machine Learning 21: 11-33

Pevzner P A 2000 Computational Molecular Biology: An Algorithmic Approach. Cambridge, MA: MIT Press1

Ravi, Vikas and Sanjay 2011 DNA Sequence Assembly using Particle Swarm Optimization. Int. J. Comput. Appl. 28(10): 33-38

Setubal J and Meidanis J 1997 Introduction to Computational Molecular Biology. Brazil: University of Campinas, ch. 4 - Fragment Assembly of DNA, 105-139

Sutton G G, White O, Adams M D and Kerlavage A R 1995 TIGR Assembler: A new tool for assembling large shotgun sequencing projects. Genome Science and Tech 1: 9-19

Yang X-S and Deb S 2009 Cuckoo search via Lévy flights. In Proc. of World Congress on Nature \& Biologically Inspired Computing, (NaBIC 2009), IEEE Publications, USA, pp. 210-214

Yang X-S and Deb S 2010 Engineering optimization by Cuckoo search. Int. J. Mathematical Modeling and Numerical Optimization 1: 330-343

Zhao Y et al 2008 An improved ant colony algorithm for DNA sequence alignment. Int. Symp. Information Sci. and Eng. 683-688

Zuwairie Ibrahim and Tri Basuki Kurniawan 2009 Implementation of an ant colony system for DNA sequence optimization. J. Artif Life Robotics 293-296 